\title{
Determinação das medidas dentárias mésio-distais em indivíduos brasileiros leucodermas com oclusão normal*
}

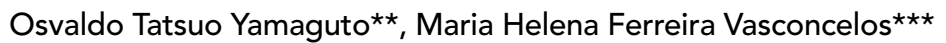

\begin{abstract}
Resumo
Em 60 modelos de gesso ortodônticos foram medidas as larguras mésio-distais dos dentes, de segundo molar a segundo molar, em ambos os arcos, utilizando um paquímetro digital modificado. Este trabalho teve como objetivos determinar o valor médio para a largura de cada dente e observar a presença de dimorfismo sexual, em indivíduos brasileiros leucodermas, com a média de idade de 16,03 anos (25 do gênero masculino e 35 do gênero feminino), não tratados ortodonticamente e portadores de oclusão normal, apresentando no mínimo quatro das seis chaves de oclusão, conforme descrito por Andrews. Os valores das médias individuais dos dentes estudados foram utilizados para a elaboração de uma tabela, correspondentes aos arcos superior e inferior.
\end{abstract}

Palavras-chave: Tamanho dentário. Oclusão normal. Medição dentária.

\section{INTRODUÇÃO}

Os modelos de estudo, integrantes da documentação ortodôntica, são importantes por possibilitarem o registro dos diâmetros mésio-distais e verticais das coroas dentárias, e das formas dos arcos superior e inferior, viabilizando a correlação destas com a face. Tais informações são fundamentais na realização de diversas análises propostas, tais como as de Bolton ${ }^{7,8}$, Moyers ${ }^{26}$, Steiner ${ }^{33}$ e Tweed $^{36}$. Estas análises, por sua vez, auxiliam a elaboração do plano de tratamento, no intuito de atingir uma oclusão ideal.

Os dentes são por natureza estruturas com formas definidas, portanto uma alteração de forma individual ou de dentes pertencentes a um grupo determinado, de posicionamento e/ou do diâmetro mésio-distal, poderá originar uma má oclusão. Assim sendo, atribuir a real importância do cálculo da discrepância do tamanho dentário entre os arcos superior e inferior, torna-se relevante para o ortodontista, no entendimento das limitações da terapia ortodôntica e conseqüente sucesso do tratamento.

Uma discrepância de tamanho dentário no arco superior ou inferior, de valores significativos, pode representar dificuldades para o tratamento, interferindo na correlação dos tamanhos dos arcos entre si, podendo levar à desarmonia entre eles. Sugere-se que este diagnóstico deva sempre ser estabelecido antes do tratamento clínico, como

* Resumo da Dissertação de Mestrado, apresentada à Universidade Metodista de São Paulo (UMESP), 2003.

\footnotetext{
** Mestre pelo programa de pós-graduação em Odontologia - Área de Concentração Ortodontia da Universidade Metodista de São Paulo (UMESP).

*** Professora do Departamento de Ortodontia da Faculdade de Odontologia - Área de Concentração Ortodontia da Universidade Metodista de São Paulo (UMESP).
} 
um dos procedimentos obrigatórios ao estabelecimento do plano de tratamento. Tais desarmonias têm sido apontadas, por vários autores, como um fator etiológico da má oclusão, dentre eles Ballard ${ }^{4}$, Bolton $^{7,8,9}$, Fields ${ }^{14}$, Sanin e Savara ${ }^{31}$.

Portanto, para se obter uma boa oclusão, os dentes devem ser de tamanhos proporcionais. Se dentes superiores grandes são combinados com dentes inferiores pequenos, como na montagem de uma prótese com tamanhos desproporcionais, não há como se chegar a uma oclusão ideal. Embora os dentes naturais se combinem muito bem na maioria dos indivíduos, aproximadamente $5 \%$ da população apresenta algum tipo de desproporção no tamanho dos dentes, segundo Profitt ${ }^{29}$, caracterizando uma discrepância de tamanho dentário.

\section{REVISÃO DE LITERATURA}

O primeiro pesquisador a registrar as médias das medidas obtidas do diâmetro mésio-distal da coroa de dentes humanos, do incisivo central ao terceiro molar, apresentando-as em forma de tabelas, foi Black ${ }^{6}$. Ballard ${ }^{4}$ também efetuou estas medições, em 500 pares de modelos de gesso com oclusão normal, nos quais os dentes foram medidos com um paquímetro e uma régua milimetrada. O dente de maior diâmetro mésio-distal foi comparado com o similar do lado oposto no mesmo arco, determinando-se a discrepância. Noventa por cento dos modelos apresentaram diferença no diâmetro mésio-distal entre os dentes dos lado esquerdo e direito, manifestada em $0,25 \mathrm{~mm}$ ou mais.

Ao definir oclusão normal, Strang ${ }^{34}$ referiu-se ao complexo constituído pelos dentes, maxila e mandíbula, e posição normal dos planos inclinados dos dentes, que em harmonia com suas bases ósseas e forças musculares, apresentam pontos de contatos proximais e inclinações axiais corretas. Emitiu uma importante opinião: para que o objetivo do tratamento ortodôntico seja alcançado, o ortodontista necessita de um conhecimento sólido, não somente dos aspectos biomecânicos relacionados à movimentação dentária, como da ma- nutenção da distância intercaninos e intermolares inferiores, além do controle das alterações faciais ocorridas durante o crescimento e desenvolvimento do indivíduo.

Ao relacionarem o apinhamento e/ou o espaçamento dos dentes com a soma de seus diâmetros mésio-distais, Moorees e $\operatorname{Reed}^{25}$ afirmaram que o apinhamento é mais comum em dentições com maiores diâmetros mésio-distais, ressaltando ainda como é difícil analisar os vários fatores capazes de influenciar no apinhamento ou espaçamento dos dentes. Na oportunidade, correlacionaram o tamanho das coroas dentárias ou o tamanho do arco dentário com a quantidade de apinhamento ou espaçamento. Assim, observaram o fato de ambas as condições serem um produto da união do tamanho dentário e do arco dentário.

Lundstrom $^{24}$ relacionou o diâmetro mésiodistal de 195 meninos e 124 meninas de 12 a 15 anos, dos dentes superiores e inferiores, pelas médias de três proporções, sendo a primeira para os incisivos e caninos, a segunda para pré-molares e primeiros molares e a terceira incluindo todos os dentes de primeiro a primeiro molar. A pesquisa demonstrou que a única associação ocorreu entre a proporção do diâmetro dos dentes e a possibilidade de apinhamento, onde arcos com dentes maiores tinham maior apinhamento, e com dentes menores apresentavam espaçamento. Não foi observado um ajuste de sobremordida ou sobressaliência, visando uma acomodação das desarmonias no índice do diâmetro entre os arcos superior e inferior. $\mathrm{O}$ autor concluiu que a extração pode ser justificada, do ponto de vista biológico, em alguns casos de severo apinhamento, pela suspeita de ser um resultado da falta de harmonia entre os fatores hereditários, relacionados aos dentes e maxilares.

Ao analisarem o tamanho mésio-distal das coroas dentárias de 51 indivíduos do gênero masculino e 50 do gênero feminino, Sanin e Savara ${ }^{31}$ verificaram alguns itens importantes. Em alguns casos com boa oclusão, o tamanho dos pré-molares 
e molares foi pequeno, com tendência a haver discrepância de tamanho dentário. Em contraposição, outros casos ilustraram várias discrepâncias de tamanho da coroa, resultando em más oclusões. O efeito dessa discrepância na oclusão pode ser minimizado pelas rotações e/ou inclinações dos dentes.

Peck e Peck ${ }^{28}$ afirmaram que os dentes são, por natureza, estrutura perfeitas. Com freqüência, alterações individuais podem, coletivamente, formar uma má oclusão. Para os autores, pelo fato de permanecer sensível a esse modo de ver, o ortodontista poderá, seguramente, aumentar seu entendimento em relação às limitações da terapia ortodôntica, podendo apreciar o valor dos procedimentos para alterar essas formas dentárias; e desta maneira obtendo sucesso no tratamento instituído.

Estudando os dados obtidos sobre a largura mésio-distal dos dentes permanentes de 162 americanos negros; metade homens, metade mulheres, Richardson e Malhotra ${ }^{30}$ verificaram que, tanto homens quanto mulheres mostravam padrão similar no tamanho dentário. Os dentes dos homens eram mais largos que os das mulheres, nas duas arcadas. Os primeiros pré-molares superiores mostraram-se mais largos que os segundos, enquanto que, na mandíbula, os segundos prémolares exibiram maior largura que os primeiros. Os primeiros molares apresentaram-se maiores que os segundos, nos dois arcos dentários, em ambos os gêneros.

Ghose e Baghdady ${ }^{19}$ verificaram que, em uma amostra de 161 indivíduos iraquianos; 78 do gênero masculino e 83 do gênero feminino, existia uma variação entre os diâmetros mésio-distais dos dentes correspondentes no lado direito e esquerdo, porém sem significância. $O$ dente de maior estabilidade foi o incisivo central inferior, e os dentes com a maior variação no tamanho foram os incisivos laterais e os primeiros molares superiores. Em geral, as medidas mésio-distais, para o gênero masculino, revelaram-se maiores do que para o gênero feminino. Os diâmetros dos dentes de iraquianos apresentavam-se maiores, ao serem comparados com grupos similares, em diferentes áreas geográficas da península árabe.

Santoro et al ${ }^{32}$ propuseram-se a estabelecer normas do diâmetro mésio-distal dos dentes de 54 pacientes, 36 do gênero masculino e 18 do gênero feminino. $\mathrm{O}$ diâmetro mésio-distal mostrou uma alta discrepância entre os dentes dos arcos superior e inferior, com o $1^{\circ}$ molar apresentando a maior variabilidade. Quanto ao dimorfismo sexual, os pacientes do gênero masculino apresentaram dentes mais largos em relação ao feminino.

Outra rápida análise para a verificação de discrepâncias no tamanho dos dentes posteriores consiste em comparar o tamanho dos segundos pré-molares superiores e inferiores. Neste caso, espera-se que tenham aproximadamente o mesmo tamanho. Uma discrepância menor que $1,5 \mathrm{~mm}$ é raramente significativa, porém, discrepâncias maiores interferem no resultado final do tratamento, devendo estar incluídas na lista de problemas ortodônticos, de acordo com Profitt ${ }^{29}$.

De posse de uma significativa amostra de pacientes com oclusão normal, este trabalho teve como objetivo efetuar as medições dos tamanhos dentários, estabelecendo a porcentagem de indivíduos com oclusão normal e dentes discrepantes, inclusive o dimorfismo sexual.

Sua importância, além da contribuição direta no esclarecimento de questões ligadas à finalização do tratamento ortodôntico, também recai sobre o fato de que na literatura não são encontrados trabalhos com todo este conteúdo de análise, principalmente no tocante à observação do critério estabelecido na obtenção da amostra.

\section{PROPOSIÇÃO}

Após a revisão da literatura concernente às discrepâncias dentárias e seus possíveis efeitos na oclusão dentária humana, este trabalho propôs:

1) Verificar a largura mésio-distal dos dentes de indivíduos com oclusão normal; e

2) Verificar a presença de dimorfismo sexual. 


\section{MATERIAL E MÉTODOS}

\section{Material}

Para a realização desta pesquisa, uma amostra de indivíduos portadores de oclusão normal foi criteriosamente selecionada pelos alunos do Curso de Pós-Graduação - Mestrado da UMESP. Entre 6.118 alunos de escolas particulares, estaduais e municipais da região do $\mathrm{ABC}$ Paulista, além de alunos do curso de Odontologia da Universidade Metodista de São Paulo, na faixa etária entre 12 e 21 anos de idade, foram selecionados 60 indivíduos, 25 do gênero masculino e 35 do gênero feminino, com idade média de 16,03 anos, e apresentavam no mínimo quatro das seis chaves de oclusão, conforme descrito por Andrews².

\section{Métodos}

\section{Seleção da amostra}

A primeira chave de oclusão foi considerada como indispensável. Os indivíduos apresentavam vedamento labial passivo e não receberam tratamento ortodôntico em nenhuma fase da dentição, que se apresentava intacta, ou com restaurações conservadoras com os segundos molares permanentes em oclusão. Funcionalmente, avaliaram-se os movimentos de protrusão e de lateralidade em ambos os lados. Verificou-se ausência de interferências oclusais posteriores durante o movimento de lateralidade direita e esquerda. Durante o fechamento, os contatos posteriores deveriam ser simultâneos e bilaterais, apresentando, também, contato nos caninos. Indivíduos apresentando diferenças habituais acima de dois milímetros entre a relação central e a de máxima intercuspidação não foram selecionados.

\section{Obtenção dos modelos}

Foram feitas duas moldagens nas arcadas superior e inferior de cada um dos 60 indivíduos, obtendo-se um modelo de estudo e outro de trabalho recortados de acordo com a técnica preconizada por Vigorito ${ }^{37}$, sem o acabamento final.

\section{Medição dos modelos}

Para a realização das medidas dentárias, modificaram-se as pontas ativas de um paquímetro digital normal, por falta no mercado de um específico para a realização da medição referida, trabalhando conjuntamente com um profissional Técnico da empresa (Norberto Mischi).

\section{Descrição do paquímetro}

Em um paquímetro digital normal fabricado pela Mitutoyo (Suzano/SP) com capacidade de $150 \mathrm{~mm}$, resolução de $0,01 \mathrm{~mm}$, modelo/código 500-144B, foi efetuada uma modificação da face de medição, substituindo as pontas ativas de um paquímetro digital normal, implantando duas placas de aço de $3 \mathrm{~mm}$ de largura e $2 \mathrm{~mm}$ de espessura, com as extremidades (pontas ativas) biseladas e rebaixadas na parte da face de medição, a fim de realizar os procedimentos de medição (Fig. 1, 2).

\section{Calibração do paquímetro}

Para que o aparelho continuasse com a mesma fidelidade, após a modificação foi feita a calibração.

\section{Descrição da medição dos dentes dos modelos}

As medições foram feitas diretamente nos modelos com o auxílio do paquímetro digital modi-

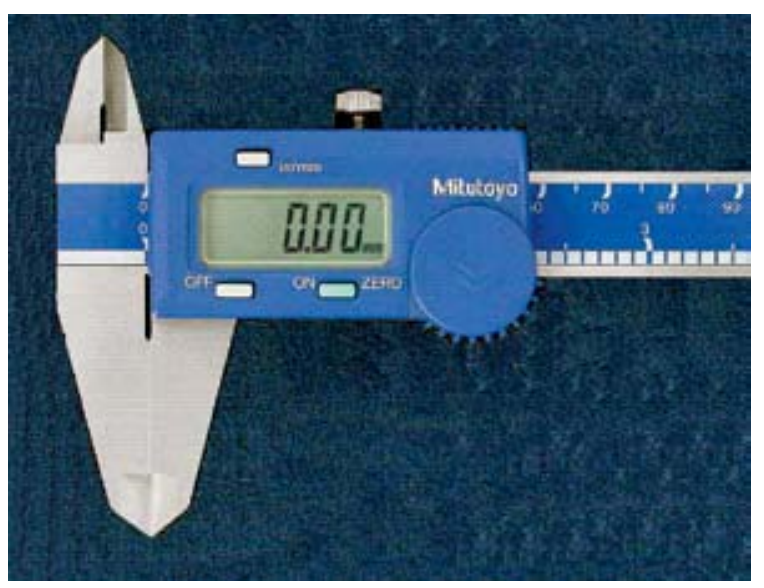

FIGURA 1 - Paquímetro digital Mitutoyo. 


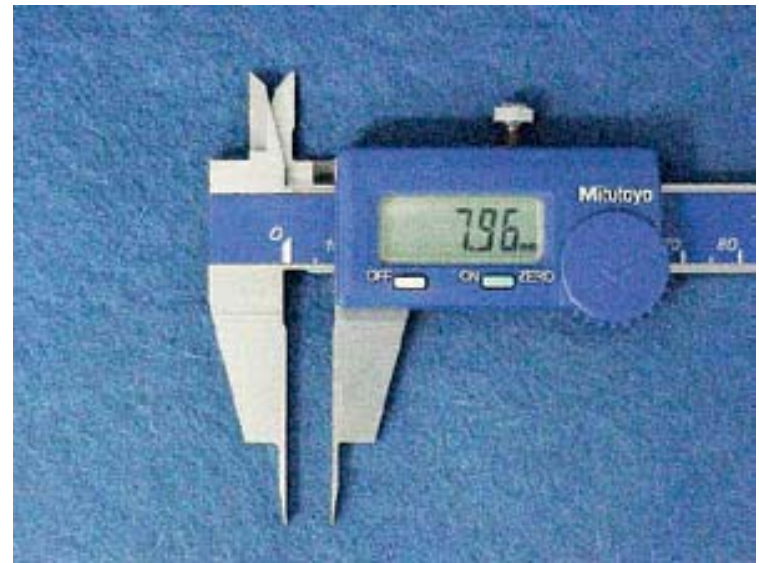

FIGURA 2 - Paquímetro digital Mitutoyo modificado.

ficado, permitindo obter valores com precisão de até $0,01 \mathrm{~mm}$, medindo-se a maior medida mésiodistal dos incisivos, caninos, pré-molares e molares, nesta seqüência.

O paquímetro foi posicionado paralelamente à superficie oclusal, pela face vestibular, perpendicularmente à superfície vestibular, medindo-se a maior distância mésio-distal do dente (Fig. 3).

\section{Método estatístico}

Os dados foram analisados por meio de tabelas e gráficos contendo valores de média, desvio padrão, valor mínimo, percentual 2,5\% e $97,5 \%$. A fim de verificar se as medidas possuíam distribuição normal, foi aplicado o teste de Kolmogorov-Smirnov, tendo sido adotado nível de significância de $5 \%$. Na comparação dos grupos masculino e feminino foi utilizado o teste $t$ de Student adotado nivel de significância de 5\%.

\section{RESULTADOS}

A tabela 1 ilustra os resultados do teste de Kolmogorov-Smirnov na verificação da normalidade. A tabela 2 demonstra os valores das médias, desvio padrão, mínimo, percentual 2,5\% e $97,5 \%$ dos 60 indivíduos. A tabela 3 compara as medidas, pelo teste $t$ de Student, entre os gêneros masculino e feminino.

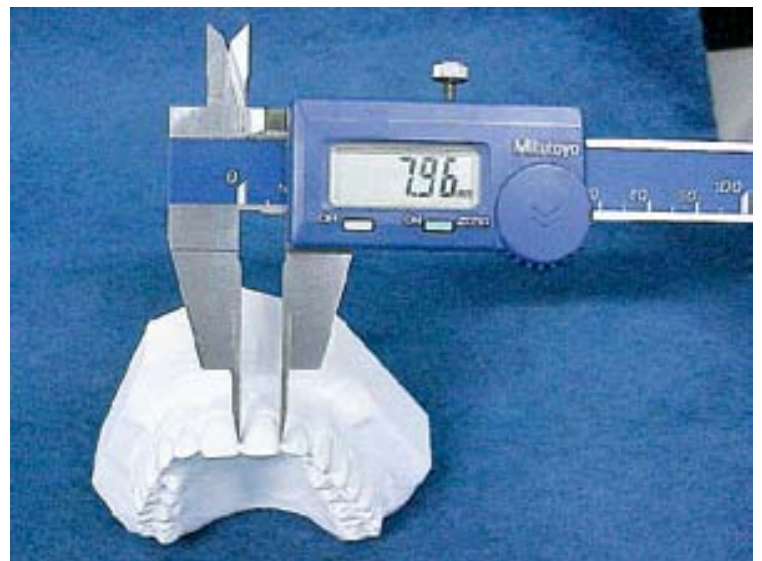

FIGURA 3 - Utilização do paquímetro modificado na medição dos dentes.

\section{DISCUSSÃO}

Sendo o diagnóstico ortodôntico um fator essencial para a elaboração de um plano de tratamento, esta pesquisa teve como objetivo principal, por meio das medições dentárias mésio-distais, obter a determinação de discrepâncias dentárias em modelos de gesso, a fim de contribuir com informações cabíveis ao diagnóstico ortodôntico. Sabemos que os ortodontistas, em sua rotina de trabalho, são capacitados para determinar as variações de tamanhos dentários e relacionar quais as influências destas nos procedimentos ortodônticos.

Em nosso estudo, as medidas foram realizadas diretamente sobre os modelos de gesso, obtidos após uma cópia fiel com alginato, representando os dentes adequadamente, como referiram Gilmore, Little ${ }^{18}$; Hunter e Prieste ${ }^{20}$, os quais obtiveram mais segurança em medidas feitas sobre modelos, do que naquelas feitas diretamente na boca do paciente.

O método usado para a medição do diâmetro mésio-distal de cada dente seguiu aquele sugerido por autores como Moorees, Reed ${ }^{25}$; Keene, Enge $^{22}$; Bushang, Dermiriian, Cadotte ${ }^{10}$ e Santoro et al. ${ }^{32}$ Segundo eles, o diâmetro mésio-distal é obtido pela maior distância entre as faces mesial e distal dos dentes, por meio de um paquímetro digital, posicionado o mais paralelo possível à superficie oclusal, pela face vestibular do dente. 
Tabela 1 - Teste de Kolmogorov-Smirnov.

\begin{tabular}{ccc}
\hline Medida & $\mathbf{d}$ & $\mathbf{p}$ \\
\hline S 7 & 0,054 & $>0,200$ \\
S 6 & 0,088 & $>0,200$ \\
S 5 & 0,055 & $>0,200$ \\
S 4 & 0,047 & $>0,200$ \\
S 3 & 0,066 & $>0,200$ \\
S 2 & 0,060 & $>0,200$ \\
S 1 & 0,049 & $>0,200$ \\
I7 & 0,070 & $>0,200$ \\
I6 & 0,045 & $>0,200$ \\
I5 & 0,090 & $>0,200$ \\
I4 & 0,084 & $>0,200$ \\
I3 & 0,073 & $>0,200$ \\
I2 & 0,069 & $>0,200$ \\
I1 & 0,069 & $>0,200$ \\
\hline
\end{tabular}

$S$ - Dentes da arcada superior.

I - Dentes da arcada inferior.

Tabela 2 - Média, desvio padrão, valor mínimo, valor máximo, percentual $2,5 \%$ e percentual $97,5 \%$ para as medidas de todos os dentes das arcadas superior e inferior dos indivíduos do gênero masculino e feminino ( $n=60)$.

\begin{tabular}{ccccccc}
\hline Medida & Média & DP & $\begin{array}{c}\text { Míni- } \\
\text { mo }\end{array}$ & $\begin{array}{c}\text { Máxi- } \\
\text { mo }\end{array}$ & P 2,5\% & P 97,5\% \\
\hline S 7 & 10,01 & 0,67 & 8,36 & 12,22 & 8,7 & 11,28 \\
S 6 & 10,11 & 0,55 & 9,03 & 11,41 & 9,16 & 11,27 \\
S 5 & 6,72 & 0,44 & 5,73 & 7,86 & 6,01 & 7,53 \\
S 4 & 7,17 & 0,45 & 6,06 & 8,23 & 6,38 & 7,53 \\
S 3 & 7,99 & 0,47 & 7,04 & 9,16 & 7,20 & 8,87 \\
S 2 & 6,85 & 0,52 & 5,39 & 8,25 & 6,02 & 7,96 \\
S 1 & 8,87 & 0,54 & 7,71 & 10,45 & 8,01 & 10,05 \\
I7 & 10,29 & 0,65 & 8,74 & 11,81 & 9,18 & 11,73 \\
I6 & 11,19 & 0,61 & 9,77 & 12,63 & 10,18 & 12,56 \\
I 5 & 7,14 & 0,44 & 6,17 & 8,31 & 6,36 & 8,14 \\
I 4 & 7,23 & 0,43 & 6,17 & 8,37 & 6,40 & 8,07 \\
I 3 & 6,93 & 0,44 & 5,90 & 8,08 & 6,15 & 7,62 \\
I 2 & 5,98 & 0,39 & 5,08 & 6,93 & 5,25 & 6,69 \\
I 1 & 5,43 & 0,33 & 4,56 & 6,54 & 4,95 & 6,05 \\
\hline
\end{tabular}

$S$ - Dentes da arcada superior.

I - Dentes da arcada inferior.

Autores como Black ${ }^{6}$ e Ballard ${ }^{4}$ foram os primeiros a registrarem os diâmetros dentários mésio-distais, obtiveram médias distintas às obtidas nesta pesqui- sa, tendo como justificativa o critério da seleção da amostra e o método de medição dos dentes.

No ano de 2000, Dall' Igna e Mendes ${ }^{11}$ realizaram um estudo no intuito de verificarem alterações nas distâncias intercaninos e intermolares inferiores em pacientes submetidos ao tratamento ortodôntico sem extrações, usarando para as medições dos modelos de estudo um paquímetro digital com precisão de $0,01 \mathrm{~mm}$ (DigitCal SI, TESA, Suíça) para o qual, devido à grande espessura de sua ponta ativa, houve necessidade de confecção de ponteiras em acrílico sobre cada extremidade, nas quais foram embutidos segmentos de fio de aço de $0,9 \mathrm{~mm}$ com pontas afiladas, para facilitar as medições e obtenção de valores mais precisos. Neste estudo, também objetivando medições dentárias precisas, sentiu-se a necessidade de aprimorar tais modificações, as quais foram conduzidas em um paquímetro digital da marca Mitutoyo, assistidas por um profissional técnico, devidamente credenciado pela empresa, no qual efetuou-se uma modificação na face de medição, substituindo as pontas ativas, implantando duas placas de aço de $3 \mathrm{~mm}$ de largura e $2 \mathrm{~m}$ de espessura, com extremidades (pontas ativas) biseladas e rebaixadas na parte da face de medição, a fim de realizar os procedimentos de medição e obtenção de valores precisos com capacidade de $150 \mathrm{~mm}$, e precisão de $0,01 \mathrm{~mm}$. Para que o aparelho continuasse com a mesma fidelidade, após a modificação realizou-se a calibração deste.

No intuito de estabelecer um coeficiente anterior e relacioná-lo à quantidade de sobremordida, Neff ${ }^{27}$ mediu o diâmetro mésio-distal dos dentes anteriores de ambos os maxilares e estabeleceu um coeficiente de valor de $1,20 \mathrm{~mm}$ a $1,22 \mathrm{~mm}$, e relatou também que uma sobremordida de $20 \%$ seria o ideal para uma oclusão normal.

Com relação ao dimorfismo sexual (Tab. 3) observou-se que: os diâmetros mésio-distais do gênero masculino são ligeiramente maiores que do gênero feminino, como também referem as pesquisas de Garn et al. ${ }^{16}$; Fastlicht ${ }^{13}$; Arya et al. ${ }^{3}$; 
Tabela 3 - Comparação das medidas dos dentes das arcadas superior e inferior, pelo teste $t$ de Student, entre os gêneros masculino e feminino.

\begin{tabular}{|c|c|c|c|c|c|c|c|}
\hline \multirow{2}{*}{ Medida } & \multicolumn{2}{|c|}{ Masculino } & \multicolumn{2}{|c|}{ Feminino } & \multirow{2}{*}{ Diferença } & \multirow{2}{*}{$\mathbf{t}$} & \multirow{2}{*}{$\mathbf{p}$} \\
\hline & Média & DP & Média & DP & & & \\
\hline S 7 & 10,35 & 0,73 & 9,77 & 0,50 & 0,58 & 5,157 & 0,000 * \\
\hline S 6 & 10,40 & 0,56 & 9,89 & 0,42 & 0,51 & 5,654 & 0,000 * \\
\hline S 5 & 6,85 & 0,41 & 6,63 & 0,43 & 0,22 & 2,858 & 0,005 * \\
\hline S 4 & 7,37 & 0,41 & 7,03 & 0,43 & 0,34 & 4,333 & 0,000 * \\
\hline S 3 & 8,34 & 0,39 & 7,75 & 0,37 & 0,59 & 8,429 & 0,000 * \\
\hline S 2 & 7,01 & 0,57 & 6,75 & 0,45 & 0,26 & 2,747 & 0,007 * \\
\hline S 1 & 9,21 & 0,52 & 8,63 & 0,42 & 0,58 & 6,780 & 0,000 * \\
\hline 17 & 10,56 & 0,58 & 10,09 & 0,62 & 0,47 & 4,248 & 0,000 * \\
\hline 16 & 11,54 & 0,53 & 10,94 & 0,54 & 0,60 & 6,058 & 0,000 * \\
\hline 15 & 7,34 & 0,43 & 6,99 & 0,38 & 0,35 & 4,621 & 0,000 * \\
\hline 14 & 7,47 & 0,42 & 7,06 & 0,35 & 0,41 & 5,734 & 0,000 * \\
\hline 13 & 7,26 & 0,36 & 6,69 & 0,33 & 0,57 & 8,871 & 0,000 * \\
\hline 12 & 6,14 & 0,43 & 5,86 & 0,31 & 0,28 & 4,095 & 0,000 * \\
\hline 11 & 5,59 & 0,34 & 5,32 & 0,28 & 0,27 & 4,639 & 0,000 * \\
\hline
\end{tabular}

* Estatisticamente significante para $<0,05$.

$S$ - Dentes da arcada superior.

I - Dentes da arcada inferior.

Richardson, Malhotra ${ }^{30}$; Doris, Bernard, Kuftinec ${ }^{12}$ e Santoro et al. ${ }^{32}$

Sabemos que, na rotina de um consultório, o ortodontista pode encontrar dificuldades ao se deparar com problemas na forma dentária.

Em sua pesquisa, Fields ${ }^{14}$ constatou que muitas condições podem contribuir para solucionar uma relativa alteração no tamanho dos dentes no arco inferior como, por exemplo, pequenos incisivos laterais superiores ou conóides. Nestes casos, movimentos dentários, em combinação com múltiplas restaurações proximais de resina composta podem ser considerados, como solução para os problemas de discrepância anterior de tamanho dentário. Em se tratando de anodontias parciais, conforme referenciadas por Garn, Lewis ${ }^{17}$; Baum, Cohen ${ }^{5}$ e Lavelle ${ }^{23}$, o problema fica agravado, já que sem uma intervenção objetivando a reposição dos dentes afetados, onde a ocorrência de anodontia de incisivo lateral é a mais comum, pode haver prejuízo do aparelho mastigatório.
Entre as formas mais comuns de prejuízo do aparelho mastigatório estão as alterações das funções e da estética, e a discrepância mais comumente encontrada seria uma provável redução mésio-distal dos dentes remanescentes, alterando com isso a relação entre os arcos superior e inferior, os movimentos funcionais de relevante importância para a obtenção de uma oclusão normal, conforme observaram Garn, Lewis ${ }^{17} \mathrm{e}$ Lavelle $^{23}$. A anodontia parcial promove espaços nos maxilares, e o fechamento e/ou manutenção tem causado polêmica entre os profissionais ortodontistas. A transformação de caninos em incisivos laterais é uma das alternativas para a solução de alguns casos, transformando o canino em incisivo lateral, por meio de desgaste das bordas incisais dos caninos quando bem indicados, e ajustados funcionalmente. As mudanças proporcionam resultados satisfatórios, segundo Turverson ${ }^{35}$; Woodworth, Sinclair, Alexander ${ }^{38}$; Furquim, Suguino, Sábio ${ }^{15}$ e Almeida et al. ${ }^{1}$ 
Após o término desta pesquisa, conclui-se que a determinação das medidas dos diâmetros mésiodistais são fundamentais no momento do diagnóstico e planejamento ortodôntico, bem como de recursos técnicos para a finalização adequada do tratamento.

\section{CONCLUSÕES}

Os resultados obtidos nesta pesquisa, permitiram as seguintes inferências:

1) A largura mésio-distal dos dentes de indivíduos leucodermas brasileiros com oclusão normal é:

\begin{tabular}{cccccccc}
\hline \multicolumn{7}{c}{ Superior } \\
\hline Medida & S7 & S6 & S 5 & S 4 & S 3 & S 2 & S 1 \\
Média & 10,01 & 10,11 & 6,72 & 7,17 & 7,99 & 6,85 & 8,87 \\
\hline \multicolumn{7}{c}{ Inferior } \\
\hline Medida & 17 & 16 & $\mid 5$ & $\mid 4$ & $\mid 3$ & | 2 & |1 \\
Média & 10,29 & 11,19 & 7,14 & 7,23 & 6,93 & 5,98 & 5,43
\end{tabular}

2) Há dimorfismo sexual em todas as distâncias mésio-distais dos dentes dos indivíduos do gênero masculino; maiores que as dos dentes dos indivíduos do gênero feminino.

\title{
Determination of mesiodistal dental measures in white brazilian individuals with
} normal occlusion

\begin{abstract}
In 60 (sixty) orthodontics models of plaster, it was measured the width mesio-distals of the teeth, from second molar to second molar, in both arches, using a modified sliding caliper. This project had as an objective to determine the average value for the width of each tooth and to observe the presence of sexual dimorphism, in white brazilians individuals, with an average age of 16.03 years old (25 male and 35 female), who were not treated orthodontically and that had normal occlusion, as it was described by Andrews. People's average values of the studied teeth were used to create a table, related to the superior and inferior arches. While analyzing the changes statistically changed, it was noticed a bigger mesio-distal width of the teeth from the male youngsters, related to the female ones.
\end{abstract}

Key words: Dental size. Normal occlusion. Dental measurement.

\section{REFERÊNCIAS}

1. ALMEIDA, R. R. et al. Tratamento ortodôntico em pacientes com agenesia dos incisivos laterais superiores: integração Ortodontia e Dentística Restauradora (cosmética). J Bras Ortodon Ortop Facial, Curitiba, n. 40, p. 280-290, jul./ago. 2002.

2. ANDREWS, L. F. The six key to normal occlusion. Am J Orthod, St. Louis, v. 62, no. 3, p. 296-309, Sept. 1972.
3. ARYA, B. S. et al. Relation of sex and occlusion to mesiodistal tooth size. Am J Orthod, St. Louis, v. 66, p. 479-486, Nov. 1974.

4. BALLARD, M. L. Asymmetry in tooth size: a factor in the etiology, diagnosis and treatment of malocclusion. Angle Orthod, Appleton, v. 14, no. 3/4, p. 67-70, July/Oct. 1944.

5. BAUM, B. J.; COHEN, M. M. Studies on agenesis in the permanent dentition. Am J Phys Anthropol, Philadelphia, v. 35 p. 125-128, July 1971 . 
6. BLACK, G. V. Descriptive anatomy of the humans teeth 4th ed. Philadelphia: S. S. White, 1902. p. 169.

7. BOLTON, W. A. Disharmony in tooth size and its relation to the analysis and treatment of malocclusion. 1952. Thesis (Master of Science in Dentistry) - University of Washington, Seatle, 1952.

8. BOLTON, W. A. Disharmony in tooth size and its relation to the analysis and treatment of malocclusion. Angle Orthod, Appleton, v. 28, no. 1, p.1-11, July 1958.

9. BOLTON, W.A. The clinical application of a tooth-size analysis. Am J Orthod, St. Louis, v. 48, no. 7, p. 504-529, July 1962.

10. BUSHANG, P. H.; DERMIRJIAN, A.; CADOTTE, L. Permanent mesiodistal tooth size of French-Canadians. J Can Dent Assoc, Montreal, v. 54, no. 6, p. 441-444, June 1988.

11. DALL' IGNA, S. M.; MENDES, A. M. Alterações nas distâncias intercaninos e intermolares inferiores em pacientes submetidos a tratamento ortodôntico sem extrações. Ortodontia Gaúcha, Porto Alegre, v.4, n.1, jan./jun. 2000.

12. DORIS, J. M.; BERNARD, B.; KUFTINEC. M.M. A biometric study of tooth size and dental crowding. Am J Orthod, St. Louis, v.79, no. 3, p. 326-35, Mar.1981.

13. FASTLICHT, J. Crowding of mandibular incisors. Am J Orthod, St. Louis, v. 58, no. 2, p.156-163, Aug. 1970.

14. FIELDS, H. W. Orthodontic restorative treatment for relative mandibular anterior excess tooth-size problems. Am J Orthod, St. Louis, v. 79, no. 2, p. 176-183, Feb. 1981.

15. FURQUIM, L. Z.; SUGUINO, R.; SÁBIO, S. S. Integração Ortodontia - Dentística no tratamento da agenesia bilateral dos incisivos superiores: Relato de um caso clínico. R Dental Press Ortodon Ortopedia Facial, Maringá, v. 2, n. 5, p. 10-33, set./out. 1997.

16. GARN, S. M. et al. Genetic control of dimorphism in tooth size. J Dent Res, Chicago, v. 46, no. 5, p. 963-972, Sept./Oct . 1967.

17. GARN, S. M.; LEWIS, A. B. Effect of agenesia on the crown size profile pattern. J Dent Res, Chicago, v. 48, no. 6, p.13-14, Nov./Dez. 1969

18. GILMORE, A.; CHARLES.; LITTLE, M. ROBERT. Mandibular incisor dimensions and crowding. Am J Orthod, St. Louis, v. 86, no. 6, p. 493-502, Dec. 1984.

19. GHOSE, L. J.; BAGHDADY, V. S. Analysis of the Iraqi dentition; mesiodistal crown diameters of permanent teeth. J Dent Res, Chicago, v. 58, no. 3, p.1047-1050, Mar. 1979.

20. HUNTER, W. S.; PRIEST, W. R. Errors and discrepancies in measurement of tooth size. J Dental Res, Chicago, v. 39, no. 2 , p. 405-414, Mar. 1960.

21. KEENE, H. J. Epidemiologic study of tooth size variability in cariesfree naval recruits. J Dental Res, Chicago, v. 50, no. 5, p.1331-345, Sept. 1971.
22. KEENE, H. J.; ENGEL, G. The mandibular dental arch, part IV: prediction and prevention of lower anterior relapse. J Dental Res, Chicago, v. 9, no. 3, p.173-179, July 1979.

23. LAVELLE, C. L. B. Maxilary and mandibular tooth size in different racial groups and in differente occlusal categories. Am J Orthod Dentofacial Orthop, St. Louis, v. 61, no. 1, p. 29-37, Jan. 1972.

24. LUNDSTROM, A. Variation of tooth size ind the etiology of malocclusion. Am J Orthod, St. Louis, v. 41, no.11, p. 872-876, Nov. 1955.

25. MOOREES, C, F. A.; REED, R. B. Biometrics of crowding and spacing of the teeth in the mandible. Am J Phys Anthropol, Philadelphia, v. 12, no. 1, p. 77-88, Mar. 1954.

26. MOYERS, R. Analysis of the dentition and occlusion. Handbook of Orthodontics. 4th ed. Chicago: Year Book, 1973. p. 351-386.

27. NEFF, C. W. Tailored occlusion with the anterior coefficient. Am J Orthod, St. Louis, v. 33, no. 5, p. 309-314, Apr. 1949.

28. PECK, S.; PECK, H. Orthodontic aspects of dental anthropology. Angle Orthod, Appleton, v. 45, no. 2, p. 95-102, Apr. 1975.

29. PROFFIT, W. R. Ortodontia contemporânea. 3. ed. Rio de Janeiro: Guanabara Koogan, 2002. p. 157-159.

30. RICHARDSON, E. R.; MALHOTRA, S. K. Mesiodistal crown dimension of the permanent dentition of American negroes. Am J Orthod, St. Louis, v. 68, no. 2, p.157-164, Aug. 1975.

31. SANIN, C.; SAVARA, B. S. An analysis of permanent mesiodistal crown size. Am J Orthod, St. Louis, v. 59, no. 5, p. 488-500, May 1971.

32. SANTORO, M. et al. Mesiodistal dimensions and tooth size discrepancy of the permanent dentition of Dominican americans. Angle Orthod, Appleton, v. 70, no. 4, p. 303-307, Aug. 2000.

33. STEINER, C. C. The use of cephalometrics as an aid to planning and assessing orthodontic treatment. Am J Orthod, St. Louis, v. 46, no. 10 , p. 721-735, Oct. 1960.

34. STRANG, R. W. The fallacy of denture expasion as a treatment procedure. Angle Orthod, Appleton, v. 19, no. 1, p. 12-22, Jan. 1949.

35. TURVERSON, D. L. Orthodontic treatment using canines in place of missing maxillary incisors. Am J Orthod, St. Louis, v. 58, no. 2, p. 109-127, Aug. 1970.

36. TWEED, C. H. The Frankfort mandibular incisor angle (FMIA) in orthodontic diagnosis treatment planing and prognosis. Angle Orthod, Appleton, v. 24, no. 3, p.131-169, July 1954.

37. VIGORITO, J. W. Documentação em clínica ortodôntica. In: INTERLANDI, S. Ortodontia: bases para a iniciação. São Paulo: Artes Médicas, 1977. p. 323-325

38. WOODWORTH, D. A.; SINCLAIR, P. M.; ALEXANDER, R. G. Bilateral congenital absence of maxilary lateral incisors: a craniofacial and dental cast analysis. Am J Orthod, St. Louis, v. 87, no. 4, p. 280-293, Apr. 1985.

39. ZAR, J. H. Bioestatistical analysis. New Jersey: Prentice Hall, 1996.
Endereço para correspondência

Osvaldo Tatsuo Yamaguto

Endereço: R. Rui barbosa, 778 - sala 61

Centro - Foz do iguaçu

CEP: 85.851-170

E-mail: ocy@uol.com.br 